\title{
Médiévales
}

Langues, Textes, Histoire

\section{Manger et boire à la mode étrangère : adoption, adaptation et rejet des pratiques festives continentales dans la Grande-Bretagne du viI ${ }^{\mathrm{e}}$ siècle}

Alban Gautier

\section{(2) OpenEdition}

Journals

Édition électronique

URL : https://journals.openedition.org/medievales/1550

DOI : $10.4000 /$ medievales. 1550

ISSN : $1777-5892$

Éditeur

Presses universitaires de Vincennes

Édition imprimée

Date de publication : 1 décembre 2006

Pagination : 37-52

ISBN : 978-2-84292-193-4

ISSN : 0751-2708

Référence électronique

Alban Gautier, « Manger et boire à la mode étrangère : adoption, adaptation et rejet des pratiques festives continentales dans la Grande-Bretagne du vıl siècle », Médiévales [En ligne], 51 | automne 2006, mis en ligne le 02 novembre 2010, consulté le 22 avril 2022. URL : http://

journals.openedition.org/medievales/1550; DOI : https://doi.org/10.4000/medievales.1550

Ce document a été généré automatiquement le 22 avril 2022.

Tous droits réservés 


\title{
Manger et boire à la mode étrangère : adoption, adaptation et rejet des pratiques festives continentales dans la Grande- Bretagne du VII ${ }^{\mathrm{e}}$ siècle
}

\author{
Alban Gautier
}

1 On lit bien souvent que la fin $\mathrm{du}_{\mathrm{VI}}{ }^{\mathrm{e}}$ et le $\mathrm{VII}^{\mathrm{e}}$ siècle marquent la réintégration de l'Angleterre à l'espace européen. La christianisation amorcée par la mission grégorienne, la reprise des contacts économiques grâce au grand commerce frison, les mariages entre dynasties anglo-saxonnes et familles franques en sont des signes évidents. Dans le même ordre d'idée, le tumulus $\mathrm{n}^{\circ} 1$ de Sutton Hoo contient un des assemblages les plus impressionnants de tout l'Occident médiéval ${ }^{1}$. En particulier, l'abondance de la vaisselle, en métal, en céramique, en bois ou en corne, vaisselle souvent (mais par uniquement) importée, fait de ce site l'un des plus importants pour la connaissance de l'Angleterre à cette époque : elle est surtout le signe de l'importance de l'activité alimentaire festive et de la prégnance de cette activité dans le système de représentations de celui qui fut enseveli à Sutton Hoo et/ou de ceux qui l'y ensevelirent. Pour les mêmes décennies, les sites d'élites des côtes occidentales de l'île ont livré d'impressionnants assemblages de céramique provenant du bassin méditerranéen. Il est évident que la vaisselle importée a joué un rôle essentiel dans les pratiques de distinction au sein des populations post-romaines, qu'elles soient anglosaxonnes ou brittoniques.

2 Mais comment aller au-delà du simple inventaire des artefacts et de la seule comparaison avec des objets semblables? Est-il possible de tirer de l'archéologie des renseignements sur l'utilisation de cette vaisselle, et éventuellement sur les activités ou rites qui pouvaient être l'occasion de cette utilisation? Peut-on par la même occasion éclairer d'une manière originale ce processus de « réintégration » de l'espace insulaire 
à l'univers culturel européen observé par de nombreux spécialistes ? De fait, l'étude de la vaisselle nous permet d'observer trois types de phénomènes ${ }^{2}$.

3 Tout d'abord, la présence de vaisselle importée peut être une indication de la vigueur de la culture exportatrice, qui devient attractive (et en général prestigieuse) pour certains groupes (en général les élites) dans les cultures avoisinantes. À l'inverse, l'absence de vaisselle importée, ou l'association systématique de vaisselle indigène avec des contextes d'élite et de prestige, peut être un signe de l'«autosuffisance symbolique » d'une culture, ou au moins de ses groupes dominants.

Deuxièmement, la manière dont la vaisselle importée est utilisée par les groupes importateurs, et les différences entre celle-ci et la manière dont les groupes exportateurs l'utilisent, sont un indice de l'adoption ou non des gestes et des valeurs qui accompagnent cette vaisselle dans son milieu d'origine : si l'on n'importe que les paraphernalia de la consommation de vin grec (cratères, louches, etc.) mais qu'on en fait un usage totalement différent (en ne mélangeant pas le vin et l'eau, par exemple), c'est un signe que les valeurs grecques de la consommation de vin ne se diffusent pas en même temps que les objets. C'est donc un signe de l'intégration plus ou moins forte des régions importatrices à l'aire culturelle exportatrice, mais aussi de la diversité des formes que peut prendre cette intégration.

5 Enfin, les différences (régionales ou sociales par exemple) entre groupes importateurs sont le signe de demandes, d'attentes différentes selon les régions et selon les groupes sociaux. Elles peuvent aussi être le signe de structures sociales ou politiques différentes. Deux groupes importateurs différents peuvent avoir deux usages très divers des mêmes cratères et coupes grecs, tous deux très éloignés de leur usage classique dans le symposion. De telles différences sont donc des moyens de pénétrer le fonctionnement mental, et en particulier les valeurs, de ces groupes, mais aussi leur fonctionnement «institutionnel»: elles montrent comment, en fonction de leurs propres exigences et de leur propre bagage culturel, ils ont utilisé de tels objets et, éventuellement, ont réinterprété à leur propre usage les valeurs qui les accompagnent.

\section{Modèles et parallèles}

6 Avant de passer à l'étude de la Grande-Bretagne, je voudrais invoquer un exemple, que je considère parallèle à notre sujet, et qui nous emmènera beaucoup plus loin, jusqu'aux Philippines du $\mathrm{X}^{\mathrm{e}}-\mathrm{XvI} \mathrm{e}^{\mathrm{e}}$ siècle, avant l'arrivée des Espagnols. On a repéré, dans l'île de Negros (au centre de l'archipel, en mer de Sulu), que la chefferie de Tanjay, tout au long de la période, importait des quantités de plus en plus importantes de porcelaine chinoise, siamoise et annamite de grande qualité, constituant de véritables collections. Celle-ci servait sans doute à des repas raffinés, car dans les zones du site de Tanjay identifiées comme habitat d'élite, l'archéologie a démontré la tenue de tels repas, incluant de grandes quantités de restes osseux, avec surtout des morceaux de choix (prédominance des têtes et des os longs) et des animaux valorisés (animaux domestiques). Or, le phénomène semble s'être emballé au $\mathrm{Xv}^{e}-\mathrm{XvI}^{\mathrm{e}}$ siècle: on a l'impression que les chefs de Tanjay se sont livrés à des festins compétitifs, et qu'ils ne se sont plus contentés d'affirmer leur puissance par l'utilisation de céramique importée. De ce phénomène, et à la lumière des rapports des premiers voyageurs espagnols, Laura Lee Junker a déduit que la société philippine de cette période était certes une société à chefferies, mais que celles-ci étaient très instables. La faible densité 
de population des îles entraînait une pénurie chronique de main-d'œuvre et obligeait les chefs à s'attacher leurs sujets par des cadeaux et des festins, tout en continuant à affirmer leur pouvoir les uns face aux autres. L'emballement du phénomène au $\mathrm{XV}^{\mathrm{e}}-\mathrm{XVI}^{\mathrm{e}}$ siècle se révèle ainsi par l'extension des trouvailles de porcelaine chinoise et de céramique locale de qualité à des zones plus « populaires » du site de Tanjay. Il est donc probable que les «nobles » de Tanjay ne mangeaient plus entre eux leurs jambons et leurs gigots, mais qu'ils en faisaient profiter des catégories moins favorisées, dont les membres repartaient avec une partie de la vaisselle de luxe utilisée ${ }^{3}$. Cet exemple nous permet donc d'analyser cette région des Philippines comme une "périphérie » du monde sino-vietnamien, mais aussi d'observer comment certaines chefferies fonctionnaient comme relais privilégiés de l'influence culturelle sino-vietnamienne, s'érigeant ainsi en "centres régionaux ». On peut enfin remarquer que les chefferies continuaient à interagir sans que des systèmes d'hégémonie formelle ne soient mis en place, comme s'il n'y avait entre elles ni « centres » ni " périphéries ».

7 Ainsi, ce cas se situe à la croisée de deux modèles anthropo-archéologiques mis en lumière, entre autres, par Colin Renfrew. Celui de l'interaction entre un noyau et sa périphérie (core-periphery interaction) est bien connu de tous ceux qui étudient des entités politiques de type impérial, et souvent utilisé par les spécialistes de la Bretagne pré-romaine et romaine. Celui de l'interaction entre entités politiques de statut similaire (peer-polity interaction) est utilisé de manière privilégiée quand ces entités politiques appartiennent à une même ère de civilisation, comme l'a fait par exemple Richard Hodges dans son étude de l'Angleterre des VIII ${ }^{\mathrm{e}}-\mathrm{X}^{\mathrm{e}}$ siècles ${ }^{4}$. Or, dans la période qui s'étend de la fin de la domination romaine à l'apparition des grands emporia de Londres, Ipswich ou Hamwic au début du VIII ${ }^{\mathrm{e}}$ siècle, la (Grande-) Bretagne semble bien, comme les Philippines de notre exemple, à la croisée de ces deux modèles. Marge d'une « mer du Nord mérovingienne », selon l'expression de Ian Wood ${ }^{5}$, le sud-est de l'île est marqué archéologiquement par un afflux d'artefacts en provenance de Gaule: en ce sens, on peut parler ici d'une participation à un système noyau-périphérie. Mais dans le même temps, les royaumes anglo-saxons et brittoniques en formation interagissent en tant qu'entités similaires, comme semblent l'indiquer les directions multilatérales des mariages dynastiques attestés dans les sources écrites. À cela il faut ajouter l'existence d'hégémonies régionales, comme celle exercée par le Gwynedd sur certains royaumes bretons et celle exercée par le Kent sur le Sud-Est au début du VII siècle, ou même plus vastes, comme celle qu'exercèrent les rois berniciens au milieu du même siècle sur la plus grande partie de l'île. 


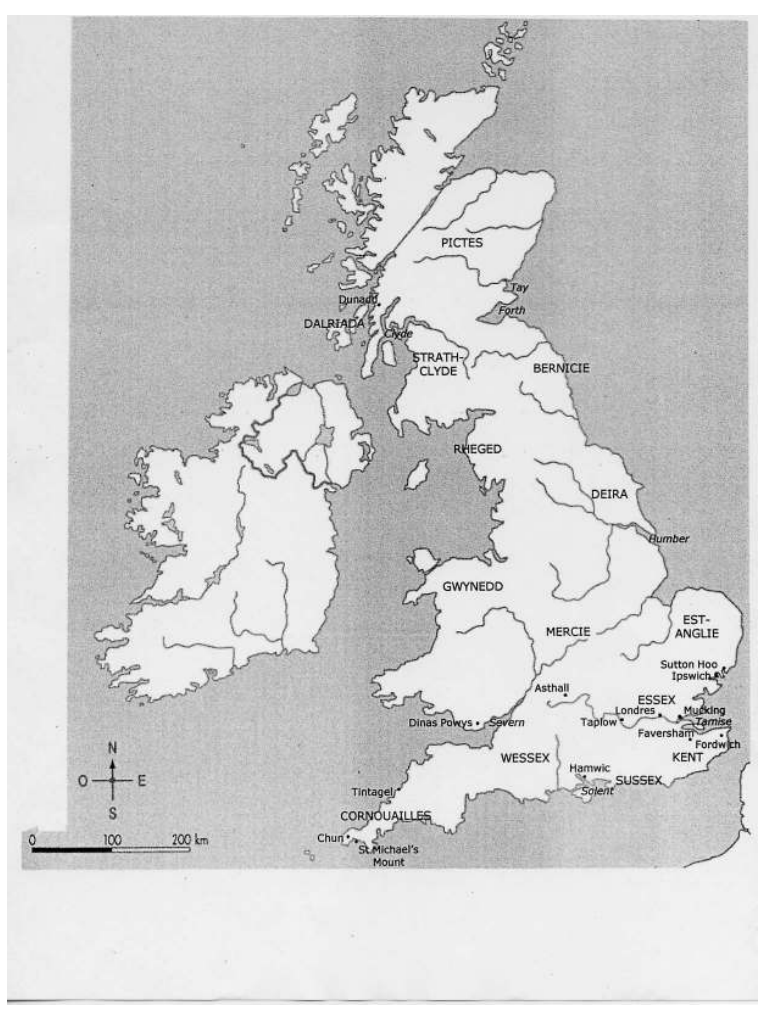

8 L'étude de ce que j'appellerai la "vaisselle importée de prestige ", en verre ou en céramique, peut-elle nous permettre d'affiner, comme dans le cas de Tanjay, ces premières impressions? La situation au milieu du $\mathrm{vl}^{\mathrm{e}}$ siècle est assez simple. Dans la moitié orientale de l'île, là où s'installe et se consolide la présence anglo-saxonne, les habitats sont quasiment acéramiques : la poterie, exclusivement montée à la main, est réservée à un usage funéraire. Dans l'Ouest au contraire, où les royautés brittoniques semblent elles aussi en voie de consolidation, les archéologues ont retrouvé dans les habitats princiers tels que Tintagel (en Cornouailles) et Dinas Powys (dans le sud du Pays de Galles) des quantités significatives de céramique en provenance du monde méditerranéen : les assemblages combinent en général une céramique de table dite de « classe A » (il s'agit de sigillées rouges fort communes dans le bassin méditerranéen) et une céramique d'emballage dite de «classe B » (ce sont essentiellement des amphores de couleur beige ayant sans doute contenu du vin ou de l'huile d'olive). Il semble bien que ces deux types de céramique étaient importés en même temps, et pour des usages concomitants, en réponse à une demande précise de la part des cours brittoniques ${ }^{6}:$ on peut donc faire l'hypothèse de l'utilisation, dans le cadre de festins, de la céramique de « classe A » comme vaisselle de prestige d'une part, et du contenu des céramiques de « classe B » comme aliments de prestige de l'autre. 


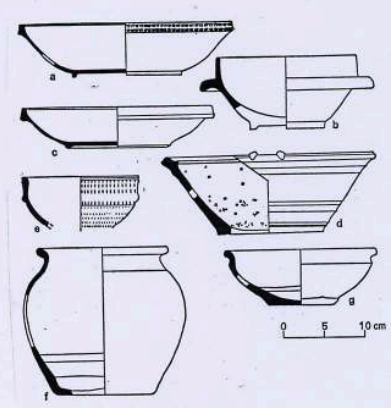

Mais la question qui nous intéresse ici est celle des évolutions observées pour la fin du $\mathrm{VI}^{\mathrm{e}}$ et le $\mathrm{VII}^{\mathrm{e}}$ siècle. Une fois exclu le "grand Nord » de l'île (le nord du monde picte est très peu touché par ces phénomènes), on peut donc dire que la Grande-Bretagne, dont toutes les régions importent, au $\mathrm{VII}^{\mathrm{e}}$ siècle, de la céramique, se divise entre des régions (l'Ouest brittonique) où de la vaisselle de prestige était déjà importée au $\mathrm{VI}^{\mathrm{e}}$ siècle, et d'autres (l'Est et le Sud-Est anglo-saxons, le Nord-Ouest scot, le Nord-Est anglo-saxon et picte) où elle ne l'était pas. J'étudierai ces deux types de régions à partir de quatre " profils» régionaux.

\section{La Cornouailles : crise ou réorientation?}

Pour le "long VII siècle", les découvertes archéologiques continuent à faire peser la balance, de manière écrasante, du côté occidental de l'île. La Cornouailles, avec les sites de Tintagel (au nord), Chun et St Michael's Mount (au sud), représente toujours une destination privilégiée de l'exportation de céramique. Certes, le commerce en provenance de la Méditerranée prend fin précisément à cette période, mais le relais semble avoir été pris par des commerçants de la Gaule de l'Ouest et du Sud-Ouest (peutêtre nantais et bordelais), qui transportent vers la Bretagne des assemblages comprenant du verre, de la vaisselle de qualité et des produits alimentaires conditionnés dans de la céramique grossière. La céramique de table est celle dite de " classe D », noire ou grise, provenant de la Basse-Loire et de la région bordelaise ${ }^{7}$. La céramique d'emballage est celle dite de "classe $\mathrm{E}$ ", grise ou blanche, provenant des Charentes, de la Basse-Loire et parfois de la Basse-Seine ${ }^{8}$. Cette dernière, longtemps désignée comme une "vaisselle de table grossière ", a été récemment réinterprétée comme une céramique d'emballage et de conditionnement de divers produits: vin, miel, fruits secs, herbes et épices (on a retrouvé des traces d'aneth et de coriandre) ${ }^{9}$. 
11 Il est donc certain que, même après le tarissement des sources méditerranéennes de cette vaisselle et de ces aliments de prestige, les élites post- ou sub-romaines éprouvaient encore le besoin d'importer des produits "exotiques" pour faire fonctionner leur système social. Celui-ci reposait sur un monopole effectif des grands centres princiers entretenant sans aucun doute des rapports exclusifs avec certains importateurs et redistribuant une partie de leurs acquisitions selon un jeu bien réglé de clientèle et de patronage. C'était le cas à Tintagel au vi ${ }^{\mathrm{e}}$ siècle, puisque l'essentiel de la céramique importée retrouvée sur ce site provenait de la région de Carthage (céramique dite de classe ARSW ou Bv) : il y avait donc là un lien privilégié entre une zone exportatrice (l'Afrique) et une cour importatrice (Tintagel). Le "prince » de Tintagel jouissait sans doute du monopole de l'importation depuis cette source particulière, et pouvait donc redistribuer à son gré ses acquisitions. Ce mécanisme a été illustré par Ken Dark avec le cas de la micro-région de Penwith. Deux centres princiers, Chun et St Michael's Mount, semblent y avoir monopolisé les importations, le second, en bord de mer, étant peut-être le «comptoir " lié au premier, dans l'intérieur des terres - une situation assez rare dans l'Ouest, où en général " comptoir » et " palais » ne sont pas séparés. Deux autres sites, moins importants, mais toujours caractérisés comme "sites d'élite", sont beaucoup moins bien fournis en céramique importée: celle-ci aurait été redistribuée depuis les centres principaux vers les centres secondaires ${ }^{10}$.

12 Ce système, s'il était efficace, a donc réussi à se perpétuer au viI ${ }^{\mathrm{e}}$ siècle grâce à des importations gauloises. On remarquera avec intérêt que, dans le système original comme dans son évolution au $\mathrm{VII}^{\mathrm{e}}$ siècle, ce que nous désignons comme vaisselle de prestige est à l'origine une vaisselle fort commune qui acquiert dans le transport son caractère prestigieux. Cette "valeur ajoutée " doit sans doute être attribuée à la distance même sur laquelle cette vaisselle est transportée et à la rareté qui, par conséquent, la caractérise, mais aussi à tout l'investissement idéologique dont peuvent faire l'objet des artefacts importés depuis ce qui restait le centre du monde romain ou, au VII ${ }^{\mathrm{e}}$ siècle, et comme un pis-aller, ce qui en tenait lieu. Ce n'est qu'au vIII ${ }^{\mathrm{e}}$ siècle que ce système d'importations et de redistributions semble s'être tari. Il est probable que c'est de cette époque qu'on peut dater véritablement la fin de la domination des modèles festifs romains dans la Bretagne occidentale: les élites sub-romaines (ou proto-galloises) cessèrent alors d'importer $\mathrm{du}$ vin et se contentèrent de produits alimentaires locaux comme la bière ou l'hydromel, se contentant aussi de la vaisselle produite localement.

On peut alors se demander si cette réorientation de l'approvisionnement est une simple conséquence du tarissement des sources d'approvisionnement ou au contraire un effet d'une crise de la demande. Le fait que les assemblages, bien que de provenance différente, reproduisent plus ou moins les mêmes schémas (de la vaisselle de table toujours accompagnée d'une céramique d'emballage) plaiderait plutôt pour la première réponse. Si c'est la demande des cours brittoniques qui a été le moteur de ces échanges au long cours, c'est la disparition de l'offre qui a forcé les importateurs à rechercher de nouvelles sources d'approvisionnement. Les commerçants de l'Ouest et du Sud-Ouest de la Gaule, qui avaient peut-être déjà joué le rôle d'intermédiaires dans les échanges $\mathrm{du} \mathrm{VI}^{\mathrm{e}}$ siècle, se trouvèrent en position « naturelle » de reprendre le flambeau.

14 Les princes cornouaillais (celui de Tintagel comme celui de Chun) ont donc maintenu au $\mathrm{VII}^{\mathrm{e}}$ siècle le système mis en place au siècle précédent. Petits centres de leurs petits 
mondes, les cours brittoniques de Cornouailles se voyaient sans doute comme des cours romaines, perpétuant les usages romains, mais on ne peut pas dire que, d'abord marges du monde byzantin, elles sont devenues marges du monde franc: en aucun cas elles ne peuvent être considérées comme les "périphéries » de ces deux espaces. Les contacts en effet étaient ponctuels et se limitaient au séjour occasionnel de commerçants, sans doute des entrepreneurs indépendants venus du bassin méditerranéen puis de l'Ouest de la Gaule. Au VII siècle surtout, le remplacement d'une source d'approvisionnement par une autre n'a pas signifié l'adoption de nouveaux comportements : au contraire, elle a été le moyen de perpétuer les anciens. L'échange compétitif qui se faisait autour de la vaisselle serait donc resté pour l'essentiel confiné à l'intérieur des principales entités politiques, ne s'étendant qu'exceptionnellement à une interaction entre entités similaires à l'intérieur de la région: la céramique d'origine africaine présente en grande quantité à Tintagel n'est attestée que dans la péninsule Sud-Ouest, et est absente du Pays de Galles ${ }^{11}$.

\section{Le Dalriada : quelle périphérie?}

Dunadd, la «capitale » des rois scots du Dalriada dans la péninsule écossaise d'Argyll, devient elle aussi au $\mathrm{VII}^{\mathrm{e}}$ siècle un centre de redistribution de la céramique importée de Gaule occidentale ${ }^{12}$. La différence entre "centre princier monopoliste " et "centres secondaires » est ici particulièrement évidente, puisque la vaisselle identifiée comme de prestige (verre, céramique de « classe $\mathrm{D}$ » et de fabriques équivalentes) se retrouve presque exclusivement à Dunadd, tandis que les nombreux petits centres de la région ont aussi livré de la céramique de "classe $\mathrm{E}$ ", c'est-à-dire des contenants témoignant de la redistribution de certaines denrées alimentaires comme le vin. Cette redistribution s'étendait d'ailleurs à d'autres régions, singulièrement les marges méridionales du pays picte, puisqu'on en a retrouvé sur l'estuaire de la Tay ${ }^{13}$.

Que déduire de ces découvertes de Dunadd et de l'Argyll ? Cette région, alors en train de devenir chrétienne (Columba meurt à Iona en 597), semble avoir repris un certain nombre d'usages en vigueur dans les régions qui ont pu servir de modèle de christianisation, mais aussi de modèle politique: le Sud-Est de l'Irlande (qui avait connu au $\mathrm{VI}^{\mathrm{e}}$ siècle les importations de céramique d'origine méditerranéenne) ou l'Ouest de la Bretagne. Il me semble ici que l'on peut véritablement parler de l'importation de pratiques en même temps que de celle d'artefacts. La mise en place d'un pouvoir nouveau dans cette région, que la tradition écrite attribue à des immigrés originaires du Nord-Est de l'Irlande, plaide pour une importation «en bloc» des pratiques religieuses, politiques, festives et sociales par une nouvelle élite ou une nouvelle dynastie, ou du moins pour l'adoption «en bloc» par les élites locales de nouvelles pratiques.

Peut-on pour autant parler du Dalriada comme d'une "périphérie» du monde brittonique? Il me semble au contraire que les contacts et les affrontements entre le Dalriada et ses voisins irlandais et brittoniques se font sur un pied d'égalité. Il vaudrait mieux parler ici d'une intégration du Dalriada à au moins deux circuits d'interaction entre entités politiques similaires : un circuit proprement scot, de part et d'autre du Canal du Nord; et un circuit nord-britannique, incluant les royaumes angles de Northumbrie, les royaumes brittoniques du Nord (Rheged, Strathclyde) et certains royaumes pictes. Il est d'ailleurs possible, comme le suggèrent certains passages de 
l'Histoire ecclésiastique de Bède, que ces deux circuits n'en aient fait qu'un : les mariages dynastiques et les nombreux séjours de membres de la dynastie bernicienne en Irlande et au Dalriada vont dans ce sens ${ }^{14}$. Il est toutefois intéressant de remarquer que cet ensemble, à l'exception des royaumes northumbriens, avait adopté les modes alimentaires de circuits plus méridionaux: ces modes d'origine romaine que nous avons repérées à Tintagel et à Chun, mais que l'on observe aussi dans de nombreux sites de part et d'autre de la mer d'Irlande. En cela, mais en cela seulement, les "circuits nordiques» dans lesquels était intégré le Dalriada peuvent être décrits comme des périphéries d'un ensemble plus vaste.

\section{Le Kent : une périphérie du monde franc?}

Cette image de continuité des choix alimentaires et, dans une certaine mesure, de décoration de la table, s'impose donc pour la moitié occidentale de l'île. $N$ 'en déplaise à certains champions de la "romanité tardive » à tout prix, elle contraste fortement avec celle qui se dégage de l'étude de sa partie orientale. Là, au contraire, les récipients en terre ne semblent pas avoir joui d'un grand prestige, ce que leur piètre qualité générale explique en partie. Il est vrai que notre vision de l'importation de vaisselle dépend essentiellement de l'archéologie funéraire : or les rites funéraires ont leurs propres logiques, et il est difficile de comparer les résultats obtenus sur cette base avec ceux obtenus à partir des sites d'habitat de l'Ouest et du Nord-Ouest.

On peut cependant affirmer que les courants commerciaux venus de la Méditerranée ou de la Gaule occidentale n'ont pas atteint le monde " anglo-saxon » au Vie-VII ${ }^{\mathrm{e}}$ siècle. En revanche, des contacts existaient en particulier avec la Rhénanie et la Belgique actuelles. Ces régions en effet exportaient du verre vers le Sud-Est de la Bretagne. Le cas des "gobelets à trompes", que les archéologues anglophones appellent "claw beakers ", est très instructif, d'autant plus que nous bénéficions à leur sujet d'une étude relativement récente de Vera Evison ${ }^{15}$. Il s'agit de très beaux verres, assez grands (ceux de Taplow mesurent environ $30 \mathrm{~cm}$ de haut), fabriqués sur le Continent (Rhénanie et actuelle Belgique) au v viècle, puis en Angleterre même au vi ${ }^{e}$ et surtout au viI ${ }^{\mathrm{e}}$ siècle. 


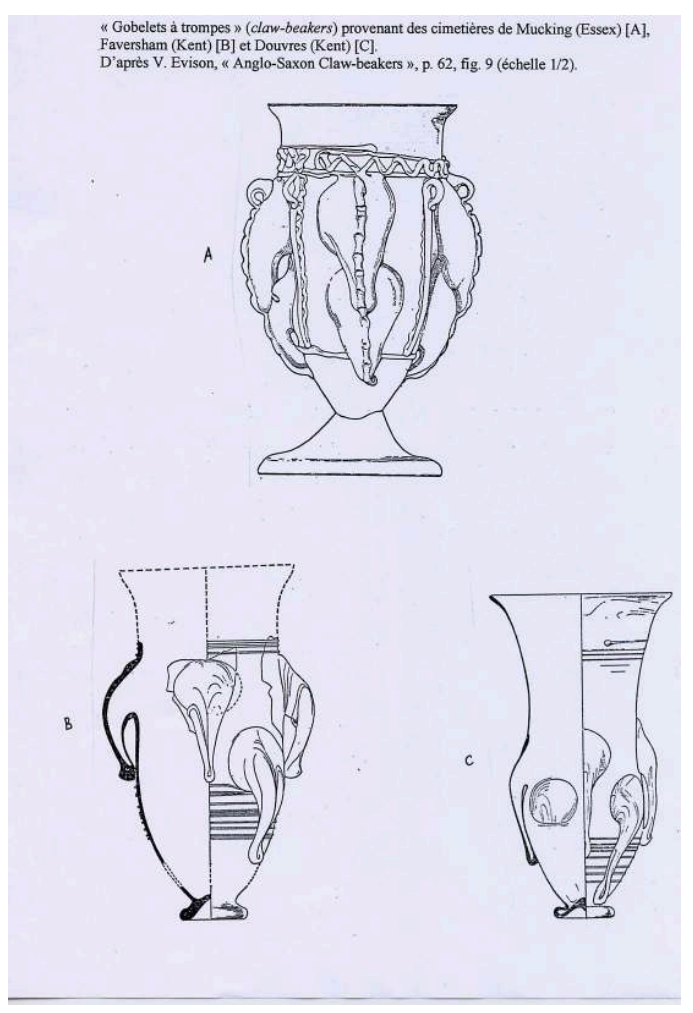

Ce que Vera Evison a montré, c'est que ces objets s'inspirent largement de modèles romains tardifs, en particulier des verres à figures appliquées (dauphins, etc.). On a d'ailleurs retrouvé dans le cimetière de Mucking un verre romain de la fin du $\mathrm{IV}^{\mathrm{e}}$ ou du début $d u v^{e}$ siècle, mais qui n'avait été enseveli que dans le cours $d u v^{e}$ siècle. Cela signifie d'une part qu'on a conservé ce verre comme objet de prestige pendant environ un siècle et demi, et d'autre part que de tels objets, encore visibles - mais aussi les verres continentaux importés dans le même temps - ont pu servir de modèles pour la fabrication d'imitations locales. Or si ces verres sont assez répandus au $\mathrm{vI}^{\mathrm{e}}$ siècle, et ce dans tout l'est de l'Angleterre (Kent, Est-Anglie, est des Midlands), ils se raréfient au VII siècle et sont confinés - en dehors du cas de Taplow - au seul Kent. D'ailleurs, deux des quatre gobelets à trompes de Taplow auraient été importés, comme beaucoup d'autres objets du tumulus, depuis le Kent.

Une explication possible de ce phénomène serait que les gobelets à trompes sont le signe de l'influence du Kent (leur centre de production principal serait d'ailleurs dans le Kent, peut-être à Faversham). Leur grande fortune dans la seconde moitié $d u \mathrm{VI}^{\mathrm{e}}$ siècle serait due à l'avancée de l'hégémonie - culturelle, voire politique puisque Bède nous autorise à le dire ${ }^{16}$ - du Kent. La seconde moitié du VI siècle correspond en effet à l'essor des Oiscingas, une dynastie originaire de l'Est du Kent, principalement sous le règne $\mathrm{d}$ 'Æthelberht, bien connu pour avoir accueilli la mission d'Augustin. Le fait que le modèle de ces gobelets soit d'origine continentale convient parfaitement à cette explication : le Kent a sans doute été la porte d'entrée principale de l'influence franque en Angleterre, et c'est en s'appuyant sur des contacts privilégiés avec le monde franc que les Oiscingas ont pu construire leur hégémonie. Le recul au VII ${ }^{\mathrm{e}}$ siècle des gobelets à trompe s'expliquerait de la même manière : les Oiscingas perdant leur influence sur le reste de l'île et devant se contenter de leur pouvoir désormais sans partage sur le Kent, 
celui-ci aurait seul conservé ce rapport de subordination/imitation qu'il entretenait depuis plusieurs décennies avec les Francs.

Cette impression est confirmée par l'étude de la céramique importée. Celle-ci est assez facile à identifier puisque, le tour de potier étant inconnu en Bretagne à cette époque, toute céramique faite au tour est nécessairement d'importation. Comme pour les gobelets à trompes, nous avons la chance de disposer d'une étude exhaustive de Vera Evison sur ce type de céramique ${ }^{17}$. Le nombre relativement restreint de récipients faits au tour retrouvés dans les tombes anglo-saxonnes témoigne sans doute de leur caractère prestigieux ${ }^{18}$. Ce qui frappe dans leur répartition, c'est la domination cette fois sans partage du Kent (seuls 16 objets sur 128 ont été retrouvés en dehors du Kent, dont 8 dans ses abords immédiats, c'est-à-dire dans la région de Londres et dans l'Essex).

"Carafes w en céramique au tour (wheel-thown pottery bottles) provenant des cimetières
Sarre (Kent) [A] et Faversham (Kent) $B$ [

Sarre (Kent) [A] et Faversham (Kent) [B].
D'apres V. Evison, A Corpus of Wheel Thrown Pottery..., p. 114, fig. 2 (échelle : 1/3).

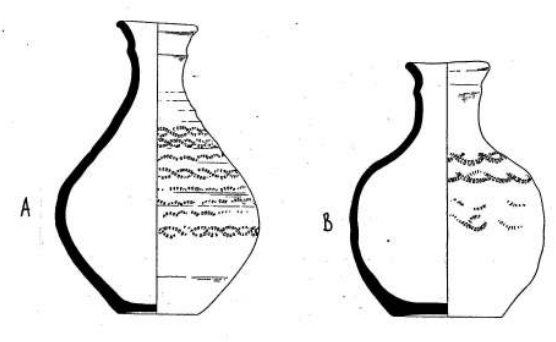

23 Les objets les plus nombreux sont des carafes ${ }^{19}$ provenant sans doute de l'actuelle région du Nord-Pas-de-Calais et de la Somme. Assez grossières, ces carafes, sur le continent, sont souvent déposées neuves dans les tombes, sans doute remplies d'un liquide - vin ou bière. En Angleterre en revanche, elles semblent avoir été utilisées pendant longtemps, sans nul doute en raison de leur rareté. Elles ont pu servir à transporter du vin, peut-être produit dans le nord de la Gaule : le fait qu'elles soient présentes en grand nombre dans le cimetière de Sarre, alors proche d'un port, irait dans ce sens ${ }^{20}$. Une fois sur place, dans le Kent, ces carafes - rapidement vidées de leur contenu - devenaient sans doute des biens de prestige, que l'on ne jetait pas mais que l'on conservait et que l'on réutilisait de nombreuses années avant de les déposer dans les tombes. De fait, les carafes d'importation retrouvées en Angleterre témoignent d'un remploi et d'une usure importants : l'une d'elles avait même été coupée en deux pour faire un bol. Ces objets ont donc été traités comme des objets rares, difficiles à se procurer. Le fait que les deux seules carafes (sur un total de 66) retrouvées hors du Kent aient été déposées dans deux des tombes les plus riches du début du vII siècle, les 
tumuli d'Asthall ${ }^{21}$ et de Sutton Hoo $\mathrm{n}^{\circ} 1^{22}$, témoigne de leur caractère précieux. Un autre indice, le fait que des potiers locaux aient (maladroitement) tenté de les reproduire à la main, ajoute à cette impression d'objets de prestige, rares et très demandés ${ }^{23}$.

Ainsi, "périphérie» de l'aire de civilisation franque, dernière roue du char mérovingien - Æthelberht n'épouse-t-il pas Berthe, fille orpheline d'un roi sans descendance masculine, "princesse dévaluée » de la dynastie mérovingienne ? - le Kent a pu se poser, sur la base de ces rapports privilégiés avec le continent, en " centre » d'une hégémonie qui couvrit sans doute tout le Sud de l'Angleterre, un "centre " qui contrôlait lui-même ses propres "périphéries ». Mais l'émergence de nouveaux centres hégémoniques en Est-Anglie, en Mercie, en Northumbrie, eut pour effet de le remettre à sa place : celle d'un simple espace " passeur ", dont l'influence ne s'étendait guère au-delà d'une périphérie immédiate. Leslie Webster a montré en effet combien la tombe de Taplow est originale dans son orientation quasiment exclusive vers le Kent: les autres tombes «riches» de la même époque (début du viI ${ }^{\mathrm{e}}$ siècle) ne semblent plus regarder uniquement dans cette direction.

\section{L'Est-Anglie : une périphérie en cours d'émancipation?}

L'absence, dans les tumuli de Sutton Hoo, de gobelets à trompe et de presque tous les types de vaisselle courants dans le Kent (la seule exception étant précisément la carafe d'origine gauloise), demande à être expliquée. Le tumulus $n^{\circ} 1$ est sans doute - les hypothèses sont nombreuses mais les spécialistes reviennent presque toujours à cette conclusion - celle du roi est-anglien Rædwald. Or, nous dit Bède, Rædwald, « même du vivant $\mathrm{d}^{\prime} Æ$ thelberht, était le chef militaire de son propre peuple ${ }^{24} »$ avant d'exercer à son tour l'imperium, c'est-à-dire un certaine hégémonie sur le Sud-Est de l'île. Il est donc possible que les Wuffingas, à savoir Rædwald et ses successeurs immédiats, aient consciemment rejeté les pratiques trop liées à l'hégémonie du Kent. Ce rejet semble confirmé par l'apostasie partielle de Rædwald, mais aussi par l'appel de son second successeur Sigeberht à Félix, un évêque burgonde, pour assurer la christianisation du royaume. Originaire du royaume franc, Félix n'était pas romain et grégorien, comme les évêques envoyés depuis Cantorbéry avec l'aval des rois oiscingas.

Or on constate que c'est précisément à cette époque que se serait développé le premier en date des grands emporia anglo-saxons, celui d'Ipswich. Un emporium qui, s'il est comparable à ceux, mieux connus, de Hamwic ou de Londres, a sans doute été, dès l'origine, une fondation royale. Si le Kent était, jusqu'au début du viI ${ }^{e}$ siècle, la porte d'entrée principale des modes et des artefacts en provenance du continent, il n'est sans doute pas innocent que ces deux événements - la fin de l'hégémonie du Kent sur l'EstAnglie et la fondation de l'emporium d'Ipswich - soient contemporains. Il s'agissait, pour les souverains est-angliens, d'assurer un contact direct avec le monde franc (et frison), c'est-à-dire avec les sources de l'initiative commerciale mais aussi des modèles et des pratiques politiques du temps. Qu'il se soit seulement agi de se dégager de l'influence du Kent, ou qu'il se soit agi également de se poser en véritables concurrents des Oiscingas, les Wuffingas d'Est-Anglie ont posé là un jalon important dans la constitution de l'Angleterre du Sud et du Sud-Est, dans son entier, comme un circuit d'interaction entre entités politiques similaires tel que l'a observé Richard Hodges. En ôtant aux Oiscingas leur quasi-monopole des relations avec les Francs, ils ont préparé le 
" grand jeu » du long VIII ${ }^{e}$ siècle entre les quatre principaux royaumes anglo-saxons du Sud (Kent, Est-Anglie, Wessex, Mercie), « grand jeu » rendu possible, entre autres, par l'accès égal de chacun des quatre royaumes aux produits d'importation par le moyen de leurs emporia respectifs (Fordwich, Ipswich, Hamwic, Londres).

Entre nos deux modèles initiaux - core-periphery interactions et peer-polity interactions on voit donc que notre analyse n'a pas su ni voulu choisir. Les quatre exemples que nous avons tenté d'analyser - Cornouailles, Dalriada, Kent, Est-Anglie - peuvent tous être analysés de diverses manières. On peut regarder certains espaces comme des « centres »: c'est en particulier le cas lorsque l'on envisage les rapports des dynasties régnantes avec leur clientèle, mais aussi quand sont attestés, comme dans le cas du Kent, des phénomènes d'hégémonie politique. On peut aussi y repérer des éléments de " circuits d'interaction ": c'est le cas lorsque des modèles sont communs à toute une région, sans que l'on puisse repérer de véritable origine à ces modèles, comme quand les royaumes de l'Ouest brittoniques reportent leur approvisionnement en vaisselle de prestige vers la Gaule de l'Ouest. Il peut encore s'agir de "périphéries » : c'est le cas lorsque ces régions, ou les circuits d'interaction dans leur entier, adoptent en bloc certaines pratiques, comme avec les « circuits nordiques » dont fait partie le Dalriada, ou quand les royaumes anglo-saxons empruntent au Kent son usage des gobelets à trompe. On peut enfin identifier des entités "autonomes": c'est le cas lorsque certaines régions rejettent un hégémonie, comme l'Est-Anglie, ou semblent fonctionner en circuit fermé, comme les petits royaumes cornouaillais. Mais, dans tous les cas, la vaisselle nous fournit une porte d'accès à l'observation du devenir des modèles continentaux dans l'île, comme si ces modèles voyageaient avec les artefacts.

En cela, la Grande-Bretagne était bien à la périphérie du continent et ce passage de l'un à l'autre, comme ceux qui ont concerné, à la même époque, les modèles politiques, les croyances religieuses ou les modes de transmission du savoir, a servi à mieux l'intégrer dans la chrétienté occidentale - une observation particulièrement valable pour les royaumes anglo-saxons, qui ont cherché à s'assurer chacun un accès direct aux sources continentales d'approvisionnement en biens de prestige. Mais en même temps, parfois adoptés, parfois rejetés, les ustensiles de vaisselle sont le plus souvent adaptés, servant à d'autres usages que ceux pour lesquels ils avaient été fabriqués. Les dynasties insulaires, particulièrement dans l'Ouest, faisaient donc preuve d'un certain particularisme, voire d'une véritable autonomie : si elles importaient de la vaisselle de prestige, c'était d'abord pour l'utiliser dans leurs propres circuits, et selon leurs propres valeurs.

\section{NOTES}

1. M. CARVER, Sutton Hoo : Burial Ground of Kings ?, Londres, 1998, surtout p. 2-51.

2. Les réflexions qui suivent me sont suggérées par les résultats obtenus par Michael Dietler dans son étude de l'usage des récipients d'origine grecque dans la Gaule pré-romaine : M. DIETLER, 
«Driven by Drink: The Role of Drinking in the Political Economy of Early Iron Age France », Journal of Anthropological Archaeology, vol. 9, 1990, p. 352-406.

3. L. L. J UNKER, "The Evolution of Ritual Feasting Systems in Prehispanic Philippine Chiefdoms ", dans M. DIETLER et B. HAYDEN dir., Feasts : Archaeological and Ethnographical Perspectives on Food, Politics and Power, Washington-Londres, 2001, p. 267-310.

4. Pour les deux modèles : C. Renfrew et P.BAHN, Archaeology: Theories, Methods and Practice, Londres, 2000, p.378-384. Sur l'utilisation de ce modèle pour la Bretagne pré-romaine et romaine: C.A. SNYDER, The Britons, Oxford, 2003, p. 25. Pour l'Angleterre anglo-saxonne: R. HoDGES, Dark Age Economics. The Origins of Towns and Trade AD 600-1000, Londres, 1982 ; R. HODGES et J. Moreland, "Power and exchange in Middle Saxon England», dans S. T. DRISColl et M.R. NIEKE dir., Power and Politics in Early Medieval Britain and Ireland, Édimbourg, 1988, p. 79-95.

5. I. N. Wood, The Merovingian North Sea, Alingsas, 1983.

6. E. CAMPBELL, «The Archaeological Evidence for External Contacts: Imports, Trade and Economy in Celtic Britain, AD 400-800", dans K. R. DARK dir., External Contact and the Economy of Late Roman and Post-Roman Britain, Woodbridge, 1996, p. 83-96. K. R. DARK, Britain and the End of the Roman Empire, Stroud, 2000, p. 125-129.

7. K. R. DARK, Britain and the End..., p. 127.

8. Ibid., p. 129.

9. E. CAMPBELL, "The Archaeological Evidence... ».

10. K. R. DARK, Britain and the End..., p. 167-168.

11. C. A. SNYDER, The Britons, p. 164-165.

12. A. LANE et E. CAMPBELl, Dunadd : An Early Dalriadic Capital, Oxford, 2000, p. 98-103.

13. Voir la thèse inédite d'E. CAMPBELL, Imported Goods in Early Medieval Celtic West, with Special Reference to Dinas Powys, University of Wales, 1991, et son résumé par A. LANE, «Trade, Gifts, and Cultural Exchange in Dark-Age Scotland ", dans B. E. CRAwFord dir., Scotland in Dark Age Europe, St John's House Papers 5, St Andrews, 1994, p. 103-115.

14. BÈDE, Historia ecclesiastica gentis anglorum $(=H E)$, B. COLGRAVE et R. A. B. MYNORS éd., Oxford, 1969 (séjour d'Oswald et Oswiu au Dalriada, mariage d'Oswiu et d'une irlandaise, séjour d'Aldfrith en Irlande).

15. V. I. Evison, « Anglo-Saxon Glass Claw-beakers », Archaeologia, t. 107, 1982, p. 43-76.

16. BÈDE, HE, I, 25.

17. V. I. EVISON, A Corpus of Wheel-Thrown Pottery in Anglo-Saxon Graves, Londres, 1979.

18. 128 récipients et fragments en tout, à comparer aux 256 verres et fragments de verre catalogués par D. B. Harden 20 ans plus tôt (V. Evison, A Corpus of Wheel-Thrown Pottery..., p. 43).

19. Le terme utilisé par les archéologues anglais est "bottle», terme trompeur. Il s'agit de carafes assez pansues, avec un goulot évasé, sans bec verseur et sans anse. Toutefois, comme des bouteilles, elles pouvaient être bouchées par de la poix et/ou de la cire.

20. V. Evison, A Corpus of Wheel-Thrown Pottery..., p. 49-50 et p. 57.

21. T. M. DiCKINSON et G. SPEAKE, "The Seventh-Century Cremation Burial in Asthall Barrow, Oxfordshire ", dans M. CARVER dir., The Age of Sutton Hoo: The Seventh Century in North-Western Europe, Woodbridge, 1992, p. 95-130, ici p. 101-102.

22. M. CARVER, Sutton Hoo..., p. 127.

23. V. Evison, A Corpus of Wheel-Thrown Pottery..., p. 18-21.

24. BÈDE, HE, II, 5. 


\section{RÉSUMÉS}

L'étude de la vaisselle d'importation (verre et céramique) offre une fenêtre privilégiée sur la compréhension des phénomènes d'interaction culturelle, économique et parfois même politique. Entre les deux modèles anthropo-archéologiques couramment utilisés (interaction entre entités politiques de statut similaire et interaction entre un noyau et sa périphérie), l'étude de quatre régions de la Grande-Bretagne du viI ${ }^{\mathrm{e}}$ siècle (Cornouailles, Dalriada, Kent, Est-Anglie) permet de mettre en évidence tout un « dégradé » de situations d'interaction, soit entre elles, soit avec le continent européen, de l'influence pure et simple à la quasi-autonomie culturelle et symbolique.

Adressing Foreign Food Habits: Adapting, Copying or Rejecting Continental Feasting Practices in 7thcentury Britain. The study of imported tableware (both glass and ceramic) can allow us to understand more deeply some cases of cultural, economic and even political interaction, for which two archaeological/anthropological models have generally been used: peer-polity and core-periphery interaction. Four areas in 7th century Britain are here considered: Cornwall, Dalriada, Kent and East Anglia. This paper argues that these regions may be seen as belonging to a whole spectrum of interaction situations, between each other and with the European Continent, ranging from mere influence to cultural and symbolic autonomy.

\section{INDEX}

Mots-clés : échanges, importation, archéologie, modèles d'interaction, consommation, GrandeBretagne, vaisselle, céramique, verre

Keywords : exchange, imports, archeology, interaction, consumption, Britain, tableware, pottery, glass

\section{AUTEUR}

\section{ALBAN GAUTIER}

Université du Littoral-Côte-d'Opale, Département d'Histoire, 34, Grande Rue, BP 751, 62321 Boulogne-sur-Mer Cedex 